\title{
RELAÇÃO DO NÍVEL DE ATIVIDADE FÍSICA COM O PICO DE FLUXO EXPIRATÓRIO MÁXIMO DOS COLABORADORES DE UMA CRECHE NO MUNICÍPIO DE RIO VERDE-GO
}

\author{
RELATION OF THE PHYSICAL ACTIVITY LEVEL WITH THE PEAK EXPIRATORY \\ FLOW OF EMPLOYEES OF A DAY CARE CENTER IN THE MUNICIPALITY OF RIO \\ VERDE-GO
}

\author{
Sonária Carlos de Sousa Gonçalves ${ }^{1}$ \\ Fernando Duarte Cabral ${ }^{2}$
}

\begin{abstract}
RESUMO: A inatividade física é um desafio para a saúde, pois tem sido um fator de risco para inúmeras doenças entre elas as cardiovasculares e pulmonares. Sabe-se que atividade física é essencial para prevenção da obesidade e reabilitação de tais disfunções. O objetivo deste trabalho é relacionar o nível de atividade física com o pico de fluxo expiratório máximo dos colaboradores de uma creche no município de Rio Verde - GO. O estudo foi realizado com uma amostra de 28 colaboradores entre 18 e 75 anos de ambos sexo, os mesmos foram submetidos a aplicação do questionário IPAQ versão curta para verificar o nível de atividade física e logo após, executaram três picos de fluxo expiratório máximo por meio do dispositivo peak flow meter. Através da análise dos resultados da presente pesquisa foi encontrada correlação positiva $(0,483)$ significativa $(\mathrm{P}=0,03 \mathrm{I})$ entre o Nível de Atividade Física e o do pico de fluxo expiratório e também foi encontrada correlação negativa $(-0,604)$ significativa $(\mathrm{P}=0,005)$ entre a Idade e o Pico de Fluxo Expiratório. Conclui-se que a prática de atividade física interfere significativamente no aumento do pico de fluxo expiratório máximo e que processo de envelhecimento fisiológico contribui para diminuição do pico de fluxo expiratório. Vale ressaltar que a prática de exercícios físico é indispensável tanto para o sistema músculo esquelético bem como para o sistema cardiorrespiratório melhorando a função pulmonar.
\end{abstract}

Palavras-chave: Atividade Física. Peak flow. Músculos Respiratórios.

ABSTRACT: Physical inactivity is a health challenge because it has been a risk factor for numerous diseases including cardiovascular and pulmonary diseases. Do you know that physical activity is essential for prevention and rehabilitation of such disorders. The objective of this study is to relate the level of physical activity to the maximum peak

\footnotetext{
${ }^{I}$ Acadêmica do $8^{\circ}$ periodo do curso de Fisioterapia da UNIBRAS Faculdade de Goiás de Rio Verde - GO. Email: sonariaejr@gmail.com.

${ }^{2}$ Professor Mestre: Fernando Duarte Cabral Unibras -Faculdade de Goiás

Coordenação: curso: Educação física Coordenação: Fisioterapia - rt: Hospital Municipal Universitário de Rio Verde - hmu. Coordenação: Fisioterapia - rt: Hospital estadual de santa Helena de Goiás - Herso. Atendimento Em Fisioterapia Home Care Email: fernandofisio2@hotmail.com.
} 
expiratory flow of the employees of a day care center in the city of Rio Verde - GO. The study was performed with a sample of 28 employees between 8 and 75 years of age, both of whom were submitted to the IPAQ short version questionnaire to verify the level of physical activity and, afterwards, performed three peaks of maximum expiratory flow through of the peak flow meter. The analysis of the results of the present study found a significant positive correlation $(0.483)(\mathrm{P}=0.03 \mathrm{I})$ between the Physical Activity Level and the peak of the expiratory flow, and a significant negative correlation (-0.604) was observed $(\mathrm{P}=0.005)$ between the Age and the Peak of Expiratory Flow. It is concluded that the practice of physical activity significantly interferes in the increase of peak peak expiratory flow and that physiological aging process decreases peak expiratory flow. It is worth emphasizing that the practice of physical exercises is indispensable for both the skeletal muscle system as well as for the cardiorespiratory system, improving pulmonary function.

keywords: Physical Activity. Peak flow. Respiratory muscles.

\section{INTRODUÇÃO}

A ventilação pulmonar corresponde ao volume de entrada e saída de ar nos pulmões durante um minuto. E para que isto aconteça é necessário a contração e relaxamento dos músculos respiratórios.

Sabe-se que o corpo humano se adapta de forma satisfatória ao meio e as condições em que vive. Desta forma quando submetido a uma carga imposta através da prática de exercício físico diário leva a adaptação do músculo esquelético, proporcionando a hipertrofia e força de tais músculos. De igual modo a atividade física interfere de forma significativa no fortalecimento da musculatura respiratória. Pois, diante do esforço físico será exigida mais força dos músculos respiratórios para que ocorra o aumento da ventilação para captar mais oxigênio.

Sendo assim, haverá benefícios em todo sistema cardiorrespiratório além do aumento da força dos músculos respiratórios há de adquirir também maior complacência pulmonar, aumento dos volumes pulmonares, da capacidade pulmonar total, bem como o pico de fluxo expiratório máximo. O pico de fluxo expiratório máximo pode ser mensurado por vários dispositivos entre eles o peak flow meter.

Conforme Bezerra e Gusmão (2010), O pico de fluxo expiratório refere-se a um dos métodos utilizados pra avaliar a função pulmonar, sendo elucidado como o maior fluxo obtido em uma expiração forçada a partir de uma inspiração completa ao nível da 
capacidade pulmonar total que mensura a força e a velocidade de saída do ar dentro dos pulmões em $1 / \mathrm{min}$.

O objetivo desta pesquisa é a relação do nível de atividade física com o pico de fluxo expiratório máximo dos colaboradores de uma creche do município de Rio VerdeGO, a fim de obter informações do quanto à atividade física pode trazer benefícios para o sistema respiratório aumentando os volumes pulmonares e melhorando a função pulmonar.

\section{METODOLOGIA}

Estudo descritivo e qualitativo que teve como amostra 20 individuos, sendo 19 do sexo feminino e um do sexo masculino. Os critérios de inclusão foram possuir idade entre I8 e 75 anos, ambos os sexos e que propuseram assinar o termo de consentimento livre e esclarecido. Já os critérios de exclusão foram idades inferior a I8 anos e superior a 75 anos, e os que não assinaram o termo de consentimento livre e esclarecido.

Os indivíduos incluídos na presente pesquisa participaram de uma coleta de dados constituída pela aplicação do questionário Internacional de Atividade Física (IPAQ) versão curta para verificar o nível de atividade física.

Logo após, foi realizado uma demonstração pelo avaliador como executar a manobra do pico de fluxo expiratório máximo a partir de uma inspiração da capacidade pulmonar total máxima, seguida por uma expiração forçada máxima, curta e explosiva através do bocal devidamente acoplada ao medidor de fluxo. Posteriormente os participantes ensaiaram uma vez no dispositivo e posteriormente sentados confortavelmente executaram três medidas sequênciais do pico de fluxo expiratório máximo por meio do dispositivo Peak Flow Meter e, assim selecionado a média máxima das três medidas para obtenção dos resultados.

Para a análise estatística os dados coletados foram tabulados no programa Excel e encaminhado para o estatístico o qual utilizou o programa Bioestat 5.o para obtenção dos resultados e o programa excel para fazer os gráficos. Foi aceito como estatisticamente significantes resultados com $\mathrm{p}<0.5$. 


\section{REFERENCIAL TEÓRICO}

\section{I Pico de fluxo expiratório máximo}

A ventilação pulmonar corresponde ao volume de entrada e saída de ar nos pulmões durante um minuto. E para que isto aconteça é necessário a contração e relaxamento dos músculos respiratórios. Sendo que os principais músculos da inspiração são o diafragma e os intercostais externos que durante a inspiração em condições de repouso permite a expansão da caixa torácica. Porém, em uma inspiração forçada exige ainda o recrutamento dos músculos acessórios que são os esternocleidomastóideos, os escalenos, o peitoral menor e o serrátil anterior. (WEST 2013).

Contudo segundo Costa (2004), a expiração ocorre de maneira passiva em repouso, correspondendo ao relaxamento dos músculos inspiratórios, pois a própria elasticidade do parênquima, somado aos gradientes intratorácicos e intrapulmonar é suficiente para produzir o mecanismo da expiração. Todavia durante a expiração forçada é necessário o trabalho muscular dos intercostais internos e dos músculos abdominais.

Para dos Santos et. al (2012), o pulmão do ser humano atinge sua função máxima aproximadamente na segunda década de vida, e a partir de aproximadamente 35 anos essa função começa decair progressivamente. $O$ enrijecimento da caixa torácica e a diminuição na complacência pulmonar refletem na diminuição da capacidade vital forçada no primeiro segundo, e ainda do fluxo expiratório forçado, entretanto há um aumento na capacidade residual funcional e no volume de reserva expiratório.

Conforme Bezerra e Gusmão (2010), O pico de fluxo expiratório refere-se a um dos métodos utilizados pra avaliar a função pulmonar, sendo elucidado como o maior fluxo obtido em uma expiração forçada a partir de uma inspiração completa ao nível da capacidade pulmonar total que mensura a força e a velocidade de saída do ar dentro dos pulmões em $1 / \mathrm{min}$.

Além disso, segundo Daniel (2018), o pico de fluxo expiratório máximo detecta o estreitamento das vias aéreas, determina e desenvolve medidas de tratamento e ainda alerta sobre a diminuição da função respiratória, o que possibilita ao paciente uma melhor verificação do estado em que se encontra. 


\subsection{Peak Flow}

O pico de fluxo expiratório é medido através do dispositivo denominado Peak Flow o qual é pequeno, portátil e econômico, sendo de fácil manejo (SANTOS,2013; FONSECA et al. 2006). Existem valores de referência internacionais para as medidas do Pico de Fluxo em relação à idade, à estatura e ao sexo. Porém, a melhor forma de avaliá-lo é sempre comparar o paciente com sua melhor medida prévia (FONSECA et al., 2006).

\subsection{Atividade física}

A inatividade física é um grande risco à saúde humana, levando milhões de pessoas no mundo a serem portadores de várias doenças como a hipertensão arterial, diabetes, doenças cardiovasculares, pulmonares entre outros. Preocupados com os grandes riscos a OMS lançou dia 4 de junho de 2018 um plano de ação global para ajudar os países a ampliar as ações para promover a atividade física a fim de reduzir comportamento sedentário e promover a saúde.

A literatura relata o quanto à atividade física é benéfica ao ser humano inclusive ao sistema cardiorrespiratório. Para Goldner (2013), os principais benefícios da prática regular de atividade física são a perda de peso; Melhorar a circulação sanguínea; Fortalecer o sistema imune; diminuir os riscos de doenças cardíacas; aumentar força e resistência dos ossos; melhorar a coordenação e equilíbrio; diminuir o estresse, ansiedade e depressão; promover uma maior interação social; melhorar a imagem corporal e a autoestima.

Segundo a Associação Médica Brasileira e o conselho federal de medicina (200I) um programa regular de exercícios deve possuir pelo menos três componentes como aeróbio, sobrecarga muscular e flexibilidade, variando a ênfase em cada um de acordo com a condição clínica e os objetivos de cada individuo, sempre contemplando a duração, intensidade e freqüência semanal. A parte aeróbica do exercício deve ser efetuada, se possível todos os dias, com duração mínima de 30 a 40.

O nível de atividade física é mensurado segundo Vespasiano et al ( 2012), através do IPAQ que é um questionário que permite estimar o tempo semanal gasto em atividades físicas de intensidade leve, moderada e vigorosa validado por doze países. 


\section{RESULTADOS}

A amostra foi composta por vinte indivíduos sendo is mulheres e I homem conforme figura I.

Figura 1- composição quanto ao sexo

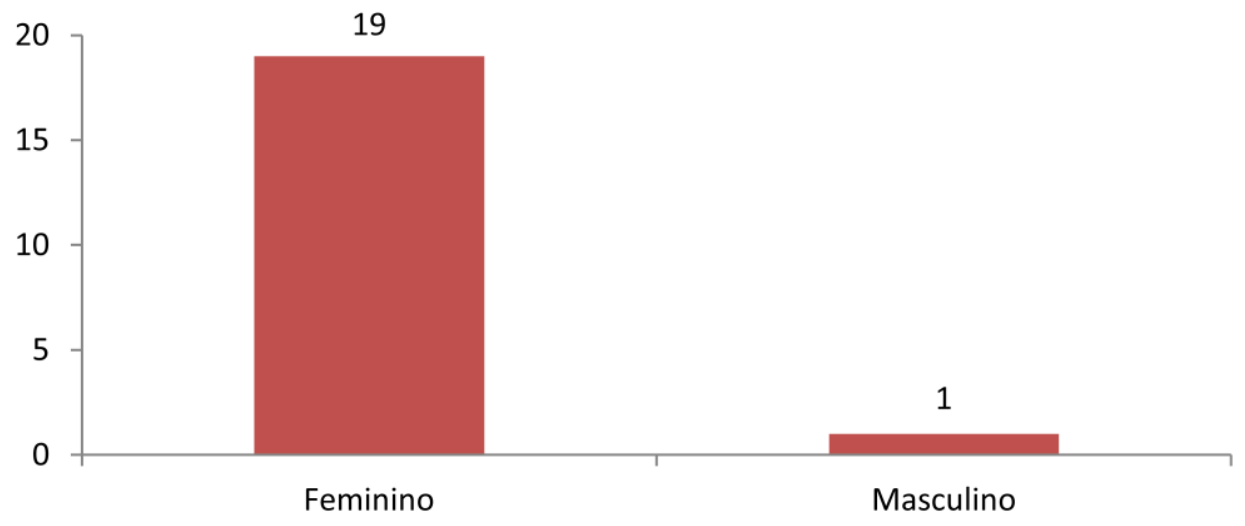

Fonte: a própria autora (2018)

A composição da amostra quanto a faixa etária é a seguinte, sendo 3 entre 19 e 3I anos, 9 entre 32 e 44 anos, 6 entre 45 e 57 anos e 2 entre 58 e 7 I anos. De acordo com figura

Figura 2- composição quanto ao sexo

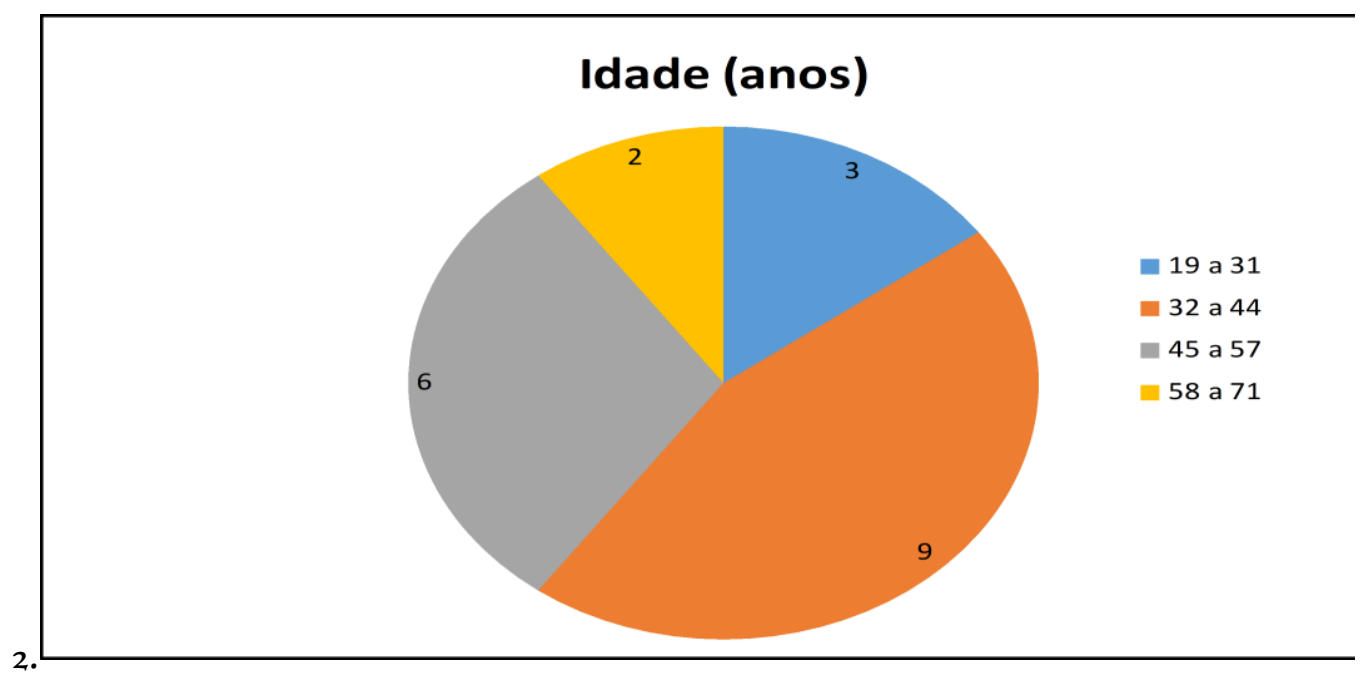

Fonte: a própria autora (2018)

A figura 3 demonstra composição da amostragem quanto a função o qual foi composta por I coordenadora, 12 recreadores, 3 cozinheiras e 4 auxiliares gerais. 
Figura 3- Composição da amostra quanto a função

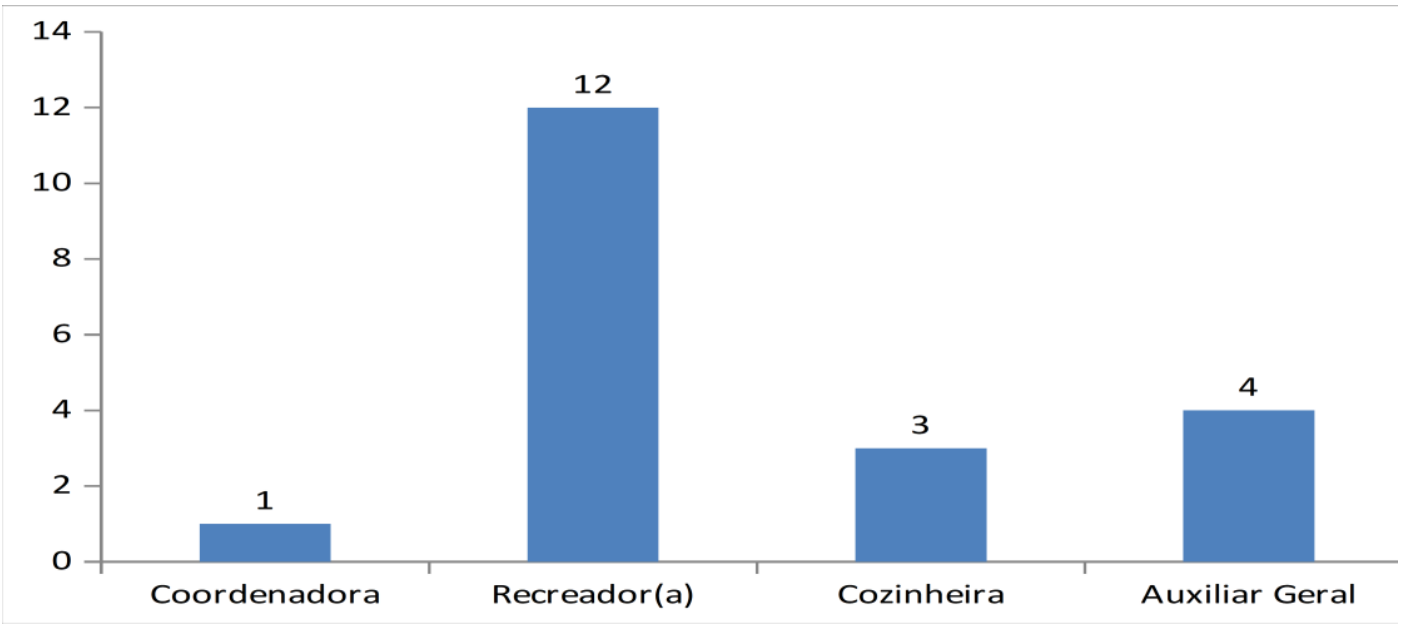

Fonte: a própria autora (2018)

A figura 4 demonstra a relação do nível de atividade conforme a faixa etária, sendo que entre a faixa etária de 19 a 3i anos mostra que teve I sedentário, I ativo e I muito ativo. A faixa etária entre 32 a 44 anos 2 são sedentários, 2 são irregularmente ativos B e 4 são irregularmente ativos A. Já entre a faixa etária de 45 a 57 anos 2 são sedentários 2 irregularmente ativo A e 2 irregularmente ativo B. Enquanto que na faixa etária de 58 a $7 \mathrm{I}$ anos I é sedentário e I é irregularmente ativo B. Os dados demonstram que 60\% dos indivíduos praticam algum tipo de atividade física.

Figura 4- Relação do nível de atividade física de acordo com a faixa etária

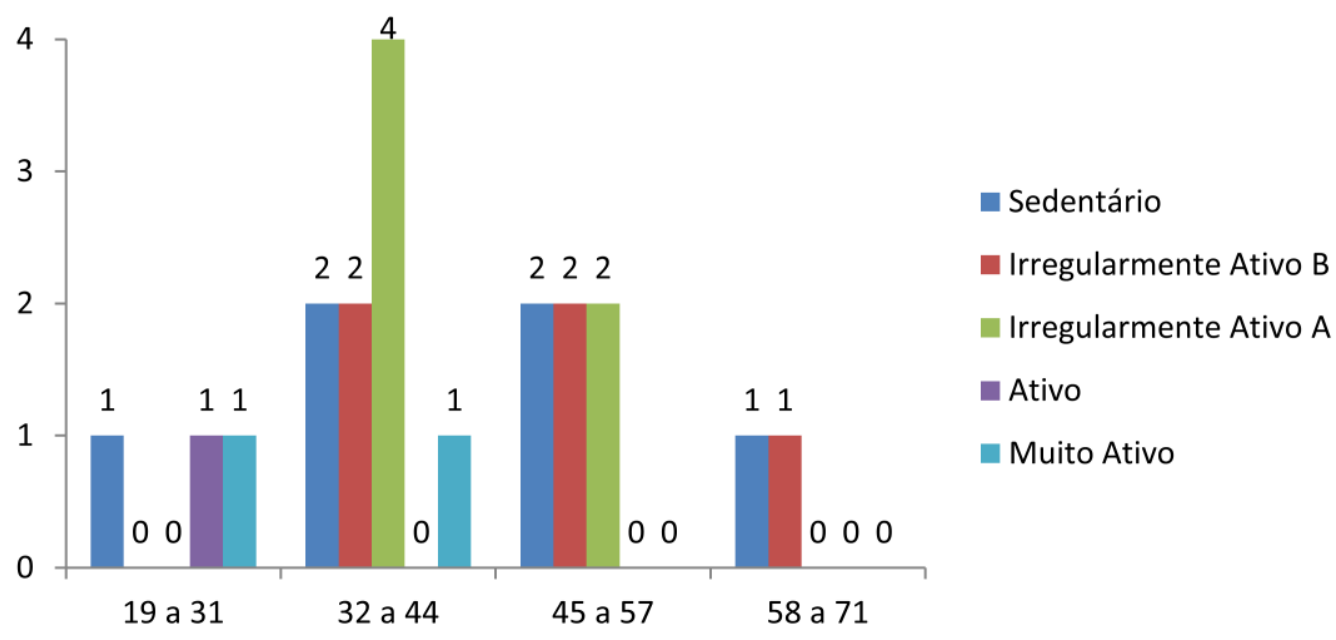

Fonte: a própria autora (2018) 
A figura 5 demonstra o nível de atividade física de acordo com a função sendo que I coordenadora é sedentária, dos 12 recreadores 2 sedentários, 3 irregularmente ativo B, 4 irregularmente ativo A, I ativo e 2 muito ativo, das 3 cozinheiras I é irregularmente ativa A e 2 são sedentárias, quanto as 4 auxiliares gerais I é sedentária, 2 é irregularmente ativa B e I é irregularmente ativa $\mathrm{A}$.

$\mathrm{Na}$ análise dos resultados na presente pesquisa não houve diferença significativa ( $\mathrm{P}$ $=0,245)$ entre os Níveis de Atividade Física de acordo com as funções, baseado no Teste Kruskall-Wallis. Apesar de que não houve uma diferença significa entre o nível de atividade física de acordo com as funções percebe-se conforme os gráficos que o grupo de recreadores foram os mais ativos ou sejam praticam algum tipo de atividade física sendo eles leve, moderado ou intenso.

A função de recreador (a) também exige um trabalho mais dinâmico de interação com as crianças com brincadeiras, jogos, esportes tornando o seu dia a dia mais ativo

Figura 5- Nível de atividade física de acordo com a função

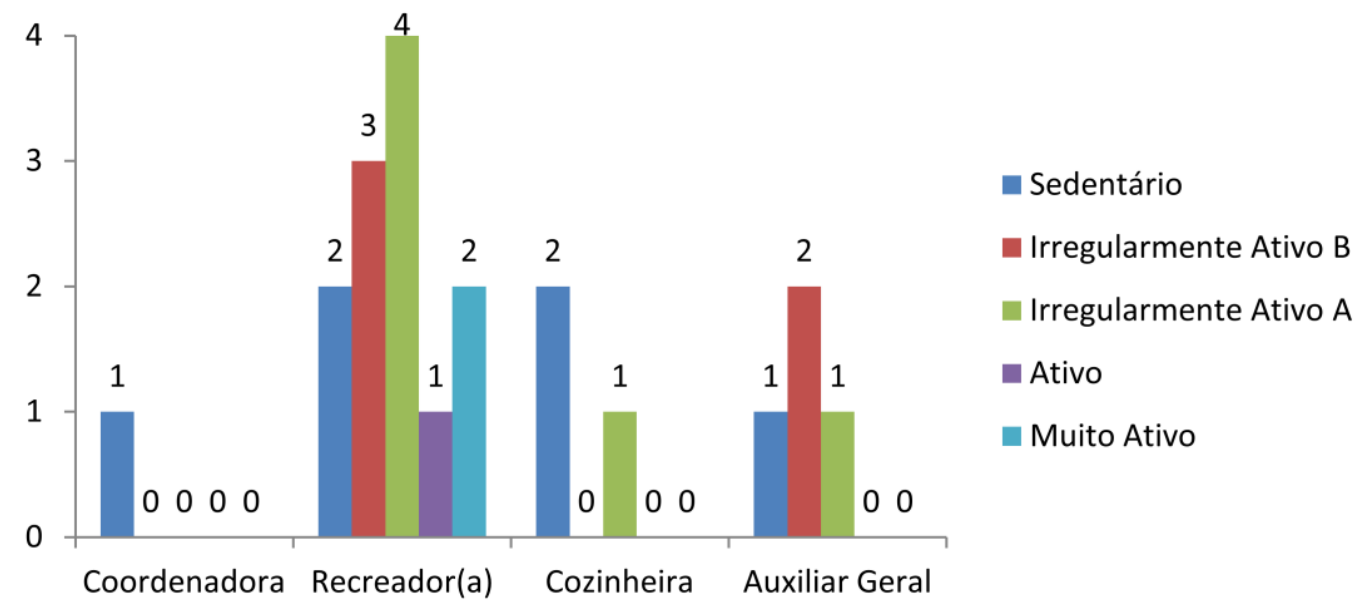

Fonte: a própria autora (2018)

A figura 6 mostra que não houve diferença significativa $(P=0,199)$ entre a média do Pico de Fluxo expiratório de acordo com as funções, baseado no teste $\mathrm{F}$ da Análise de Variância. Porém, nota-se que os recreadores obtiveram a média do pico de fluxo expiratório de 373,06 m/1 maior do que as demais funções. Isso pode ser devido ser o grupo mais ativo da amostra conforme figura 5, embora ainda esteja abaixo do nível ideal. 
Figura 6 - Relação do pico de fluxo expiratório de acordo com a função

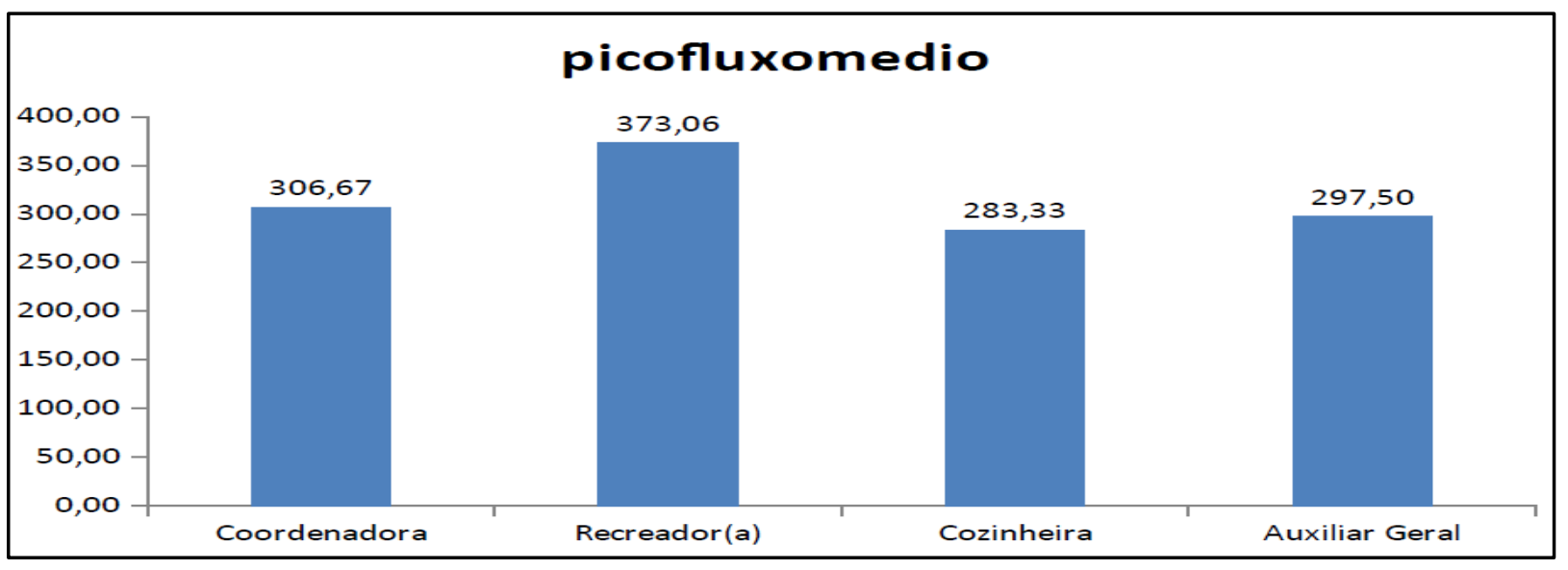

Fonte: a própria autora (2018)

Enquanto que os valores detectados através da avaliação do fluxo expiratório máximo realizado com o dispositivo Peak Flow Meter, foi encontrada correlação positiva $(0,483)$ significativa $(\mathrm{P}=\mathrm{o}, 03 \mathrm{I})$ entre o Nível de Atividade Física e o Pico de Fluxo Expiratório, baseado no Teste t. Conforme demonstrado na figura 7. Também foi encontrada correlação negativa $(-0,604)$ significativa $(P=0,005)$ entre a Idade e o Pico de Fluxo Expiratório, baseado no Teste t. Tabela 7. E ainda em conformidade com os dados, não foi encontrada correlação significativa $(\mathrm{P}=0,135)$ entre a Idade e o Nível de Atividade Física, baseado no Teste t. Figura 7.

Tabela I- correlações entre a idade e o pico de fluxo expiratório/ Atividade Física e a idade/ idade e pico de fluxo expiratório.

\begin{tabular}{|c|c|c|c|c|c|}
\hline \multicolumn{4}{|l|}{ Correlações } & \multirow{2}{*}{$\begin{array}{l}\text { Idade } \\
\begin{array}{r}-0,346\end{array}\end{array}$} & \multirow{2}{*}{ 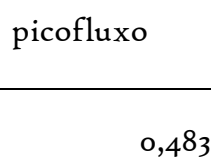 } \\
\hline \multirow{6}{*}{$\begin{array}{r}\text { Rô } \\
\text { Spearman }\end{array}$} & \multirow{6}{*}{ de } & \multirow{3}{*}{ ativ_fisica } & Correlações de coeficiente & & \\
\hline & & & Sig. (2 extremidades) & 0,135 & 0,031 \\
\hline & & & $\mathrm{N}$ & 20 & 20 \\
\hline & & \multirow{3}{*}{ picofluxomedio } & Correlações de coeficiente & $-0,604$ & \\
\hline & & & Sig. (2 extremidades) & 0,005 & \\
\hline & & & $\mathrm{N}$ & 20 & \\
\hline
\end{tabular}

Fonte: a própria autora (2018) 


\section{DISCUSSÃO}

Segundo os dados desta pesquisa, embora não tenha obtido uma diferença significativa $(\mathrm{P}=0,245)$ entre os Níveis de Atividade Física de acordo com as funções. E também, não foi encontrada correlação significativa $(P=0,199)$ entre o Pico de Fluxo expiratório de acordo com as funções. Nota - se que a amostra composta por recreadores foram os mais ativos e também obtiveram o maior pico de fluxo expiratório da amostra.

Isso pode ser em virtude de que além de praticar algum tipo de atividade física, a função de recreador (a) exige um trabalho mais dinâmico com as crianças com brincadeiras ativas como pega-pega, pique esconde e de rodas, embora esteja abaixo do nível dentro da normalidade.

Marins e Costa (2016) afirma que os exercícios de recreação, materializados através do brinquedo, da brincadeira e do jogo, influenciam tanto os alunos como o profissional de educação infantil. O professor muda seu cotidiano profissional com novas propostas de trabalho, diversificando suas aulas e crescendo como educador.

Outro resultado deste estudo atesta que houve correlação positiva $(0,483)$ significativa $(\mathrm{P}=\mathrm{o}, \mathrm{03I})$ entre o nível de atividade física e a média do pico de fluxo expiratório máximo, baseado no Teste t. Conforme figura 7. Este fato ocorre em razão de que quanto maior for o nível de atividade física mais elevado será também o pico de fluxo expiratório apesar de que os participantes não conseguiram atingir o pico de fluxo expiratório ideal.

E este resultado vai de encontro com um estudo realizado por Nascimento et al. (2015), que avaliaram o pico de fluxo expiratório de 12 indivíduos, os pesquisadores observaram que após 24 sessões de exercícios aeróbicos e resistidos houve aumento do fluxo expiratório nos participantes dos dois grupos analisados, embora, nem todos voluntários conseguissem obter a média do fluxo expiratório ideal, possivelmente devido a falta de regularidade na frequência do cumprimento do protocolo de exercícios.

Em outro estudo realizado por Oliveira et. al (2012) sobre a análise da função respiratória em praticantes de atividade física e indivíduos sedentários composto por 22 indivíduos cada grupo, os valores obtidos em relação ao Pico de Fluxo expiratório pelo grupo de praticantes de atividade física foram maiores em relação ao grupo de indivíduos 
sedentários $(p=0,966)$, porém os valores foram inferiores aos parâmetros de normalidade situa-se em torno de $586 \mathrm{l} / \mathrm{min}$, segundo (ALMEIDA et al 2009).

Ainda em conformidade com a análise de Bessine et. al (2014) em sua pesquisa sobre o pico de fluxo expiratório em adultos praticantes de atividade física e adultos sedentários, composta por 98 indivíduos, divididos em dois grupos de 49 integrantes cada, sendo que o Grupo A: ativos e o Grupo B; constatou que o grupo A de ativos apresentaram valores favoráveis estatisticamente significativos comparado ao grupo de sedentários.

Os dados obtidos por SILVA et al., (2010) que avaliaram o pico de fluxo expiratório de 65 mulheres sedentárias com idade entre 20 e 35 anos, no qual foi observado uma redução no fluxo expiratório, no volume expiratório forçado no primeiro segundo e na capacidade vital em relação aos valores normais, levando à diminuição do pico de fluxo expiratório, em decorrência da redução do volume expirado.

Outro resultado encontrada nesta pesquisa foi a correlação negativa (-o,604) significativa $(\mathrm{P}=0,005)$ entre a idade e a média do pico de fluxo expiratório máximo, baseado no Teste t. Figura 7. O mesmo confirma que há uma tendência de que quanto maior for a idade menor será o pico de fluxo expiratório. Isso ocorre devido ao processo de envelhecimento fisiológico que promove diversas alterações no organismo, levando a uma redução ou perda da massa muscular e consequentemente da força tanto dos músculos esqueléticos como também dos músculos respiratórios, acarretando consequentemente em uma rigidez na caixa torácica.(BRITTO et al 2005)

Tal perda acarreta no declínio dos valores da capacidade vital forçada do volume expiratório forçado no primeiro segundo e do fluxo expiratório forçado, bem como aumento da capacidade residual funcional e do volume de reserva expiratório. (RUIVO et.al 2009). Estes dois últimos eventos, por sua vez, relacionam-se com a redução do recolhimento elástico pulmonar e com a diminuição da complacência da caixa torácica. Tais alterações, associadas à redução da força muscular, podem levar à redução do pico de fluxo expiratório. (FREITAS et. al 2010, ANDRADE et. al 2014).

No estudo de Ruivo et al (2009) sobre efeito do envelhecimento cronológico na função pulmonar comparando a função respiratória entre adultos e idosos saudáveis. Os resultados mostraram que houve diferença do padrão respiratório entre jovens adultos e idosos saudáveis, sugerindo que a função pulmonar é influenciada pelo envelhecimento 
cronológico. Em ambos os gêneros, os indivíduos idosos apresentaram valores espirométricos mais baixos do que os indivíduos adultos, sendo esta diferença maior no gênero feminino.

A pesquisa de Andrade et al (2015) que realizou a análise comparativa de pico de fluxo expiratório de 86 idosos praticantes de atividade física e sedentários constatou que houve relação estatisticamente significativa da prática de atividade física versus Pico de Fluxo Expiratório ( $\mathrm{p}=0,0004)$ comparado a amostra de sedentários

Carneiro (2017), através da sua pesquisa sobre avaliação da função pulmonar e da capacidade funcional de um grupo de idosos da cidade de Uberlândia-MG. Concluiu que a prática de atividade física foi capaz de influenciar na função pulmonar e a capacidade funcional desse grupo de idosos.

\section{CONCLUSÃO}

De acordo com os resultados supracitados conclui-se que a prática de atividade física interfere significativamente no aumento do pico de fluxo expiratório máximo. E que o processo de envelhecimento fisiológico promove alterações também no sistema respiratório acarretando em uma rigidez da caixa torácica e uma redução do recolhimento elástico pulmonar o qual contribui para a diminuição do pico de fluxo expiratório. Por isso vale ressaltar que a prática de exercícios físico é indispensável para o sistema músculo esquelético como também para o sistema cardiorrespiratório e que traz benefícios para crianças, jovens, adultos e idosos, melhorando a função pulmonar e diminuindo os riscos de doenças. Espera se que estes dados sirvam para estimular e sensibilizar a população sobre a importância da atividade física bem como os seus benefícios à saúde humana.

\section{REFERÊNCIAS}

ANDRADE, F. S. S. D. et al. análise comparativa do pico de fluxo expiratório entre idosos sedentários e praticantes de atividade física. Revista geriatria \& gerontologia. 2015

BASSINE, S. F, MACEDO F. A. SILVA J. J. Análise do pico de fluxo expiratório em atudos praticantes de atividade fisica e adultos sedentários. Revista Científica Indexada 
Linkanis Júnior. v.4, n.3, julho/setembro 2014.

BENEDETTI, Tânia R. Bertoldo; ANTUNES, Priscilla de Cesaro ; RODRIGUEZ, Ciro Romélio. Reprodutibilidade e validade do questionário internacional de atividade física (IPAQ) em homens idosos .RevBrasMed Esporte _ Vol. I3, № I - Jan/Fev, 2007.

BEZERRA; G. K. A.; GUSMAO, A. Q. L. P. Efeitos da manobra de aumento do fluxo expiratoriosobre o pico de fluxo expiratorio em individuos sadios. Revista Brasileira de Ciências da Saúde, v.I4, n.2, Paraiba, 2010. p.13-20.

Brasil. Ministério da Saúde. Secretaria de Vigilância em Saúde. Política nacional de promoção da saúde / Ministério da Saúde, Secretaria de Atenção à Saúde. - Brasília: Ministério da Saúde. Brasília: Ministério da Saúde; 2006.

BRITTO, R.R.; VIEIRA, D.S.R.; RODRIGUES, J.M.; PRADO,L.F.; PARREIRA,V.F. Comparação do padrão respiratório entre adultos e idosos saudáveis. Rev. Bras. 452 Fisioter.2005 9(3): 28I-287.

CARNEIRO, Jéssica Cristina Borges. Avaliação da função pulmonar e da capacidade funcional de um grupo de idosos da cidade de Uberlândia - MG. 2017. 32 f. Trabalho de Conclusão de Curso (Graduação em Fisioterapia) - Universidade Federal de Uberlândia, Uberlândia, 2017.

COSTA Dirceu. Fisioterapia Respiratória Básica. Editora Atheneu, 5 ed. São Paulo, 2004.

DANIEL. Medidas de pico de fluxo expiratório máximo. Disponível em < https://fisioterapeutadaniel.webnode.com.br/news/medidas-de-pico-de-fluxoexpiratorio-maximo/>. Acesso em: 04 de setembro de 2018.

FONSECA ACCF. et al. Pico do fluxo expiratório no acompanhamento de crianças asmáticas. J Pediatr (Rio J). 2006;82(6):465. 
MACHADO, Maria da Glória Rodrigues. Anatomia e função dos músculos respiratórios. Guanabara Koogan, Rio de Janeiro, 2008.

MARINS, Danielle Stéfane de; COSTA, Celia Regina Bernardes - Recreação Escolar: o brinquedo a brincadeira e o jogo na educação da infância. Revista Científica Multidisciplinar Núcleo do Conhecimento, Ano I. Vol. Io pp. 05-24. ISSN.2448-0959. Ago/2016

MORA, Cintia Teixeira Rossato et. al. Força muscular respiratória e pico de fluxo expiratório em jovens sedentários usuários de narguilé. J Health Sci Inst. 2018; 36(2): 141-5.

MINISTÉRIO DA SAÚDE. Dia mundial da atividade física-movimentar o corpo é fundamental para saúde. Disponível em <www.blog.saude.gov.br/promocao-dasaude/50853-dia-mundial-da-atividade-fisica-movimentar-o-corpo-e-fundamental-para-asaudehtml.html>. Acesso em 29 de maio de 2018.

NASCIMENTO, Miakelle Araujo, SANTOS, Gisélia Cícera dos, Freire Rosimari de Faria. Avaliação do Peak Flow antes e após exercícios. Ciências Biológicas e da Saúde. Maceió, v. 2, n.3, p.II-20, maio de 2015.

OLIVEIRA, Maria Valéria Vieira de et. al. Análise comparativa da função respiratória em praticantes de atividade física e indivíduos sedentários. Eciclopedia Bioesfera, Centro Científico Conhecer, v.8, n.15; p.1920. Goiania, nov/2012.

SANTOS, T. C. dos; TRAVENSOLO, C. F. de. Comparison between respiratory muscle strength in sedentary and active elderly. A Transversal Study. 2012.

SILVA, B. G.; SOUZA, F. S. P.; FORTI, E. M. P. Avaliação do Pico de Fluxo Expiratório em Obesas Mórbidas. 8 Congresso de Pós-Graduação. Desafios da Educação Superior na agenda do novo Milênio, 2010. Disponível em 
http://www.paulomargotto.com.br/documentos/pico de fluxo expiratorio.ppt. Acesso em: 19/10/2018.

VESPESIANO Bruno de Souza et al. A utilização do Questionario Internacional de Atividade Fisica (IPAQ) como ferramenta diagnostica do nível de aptidão física: uma revisão no Brasil. Saúde em Revista. Piracicaba, v.12, n.32,p.49-54, set-dez.2012.

WEST, John Burnard. Fisiologia respiratória: princípios básicos. 9. ed. Porto Alegre: Artmed, 2013. 